\title{
WEYL QUANTIZATION AND A SYMBOL CALCULUS FOR ABELIAN GROUPS
}

\author{
N. J. WILDBERGER \\ (Received 8 February 2003; revised 20 October 2003) \\ Communicated by A. H. Dooley
}

\begin{abstract}
We develop a notion of a $*$-product on a general abelian group, establish a Weyl calculus for operators on the group and connect these with the representation theory of an associated Heisenberg group. This can all be viewed as a generalization of the familiar theory for $\mathbb{R}$. A symplectic group is introduced and a connection with the classical Cayley transform is established. Our main application is to finite groups, where consideration of the symbol calculus for the cyclic groups provides an interesting alternative to the usual matrix form for linear transformations. This leads to a new basis for $\operatorname{sl}(n)$ and a decomposition of this Lie algebra into a sum of Cartan subalgebras.
\end{abstract}

2000 Mathematics subject classification: primary 44A45 17B05 11F27.

\section{Introduction}

In this paper we show that a certain circle of ideas related to harmonic analysis on $\mathbb{R}$ has a natural generalization to any locally compact abelian group satisfying a certain parity requirement. The topics with which we are concerned are:

(1) the Weyl calculus for pseudo-differential operators on $\mathbb{R}$ (see for example Hormander [5]);

(2) the Moyal product, or $*$-product, for functions on $T^{*} \mathbb{R}$ (see Bayen et al. [2], Moyal [9]); and

(3) the convolution structure on the Heisenberg group.

The close connection between these subjects has been described in Howe [6, 7], Grossman, Loupias and Stein [4], Folland [3] and Lion and Vergne [8]. We wish here

(C) 2005 Australian Mathematical Society $1446-7887 / 05 \$ A 2.00+0.00$ 
to point out that these three theories can be built around any locally compact abelian group subject to a certain parity condition. This parity condition is satisfied by $\mathbb{R}$ but not by the circle group, which is an explanation for why there is no good symbol calculus for the latter. More generally, the crucial condition is that multiplication by 2 ought to be an automorphism of the group. For a finite abelian group, this simply requires that the order of the group be odd. The significance of this factor 2 was already mentioned in Howe $[6,7]$. For quite another approach using Kac algebras, see Aldrovandi and Saeger [1].

To avoid technicalities, we restrict our attention to the case of $G$ a finite abelian group; since our formulae hold in general however, we have used integrals instead of sums throughout.

We begin by considering the group $\widetilde{G}=G \times \widehat{G}$ (following Weil [10]) and its unique Haar measure. We show there is a canonical algebra structure on the space of functions on $\widetilde{G}$ which is given by an integral formula similar to one which may be used to define the Moyal product on $T^{*} \mathbb{R}$.

Furthermore, if $|G|=n$ is odd (which we henceforth assume) then there is a canonical identification of such functions as symbols of operators on $C(G)$ so, that this algebra structure corresponds to operator multiplication.

This shows that abstractly $C(\widetilde{G})$ is isomorphic to $M(n, \mathbb{C})$. Subgroups of $\widetilde{G}$ are shown to naturally lead to subalgebras of $M(n, \mathbb{C})$.

The above algebra structure is related to the representation theory of an associated Heisenberg group which we define. We study the associated oscillator representation of the symplectic group which arises as 'quadratic' symmetries of $C(\widetilde{G})$ and show how the familiar Cayley transform arises in a natural way when we derive formulae for this representation. We are here combining well known ideas from the special case $G=\mathbb{R}$ (as found, say, in Howe [6] or Lion and Vergne [8]) with the general approach of Weil [10].

It is perhaps useful to point out the direction of the applications we have in mind. The idea of representing a linear transformation of $\mathbb{R}^{n}$ by a matrix with respect to an ordered basis permeates much of modern mathematics. This is simply one convenient way of encoding $n^{2}$ bits of information and corresponds to viewing the matrix as a function on $\mathbb{Z}_{n} \times \mathbb{Z}_{n}$. However in infinite dimensional functional analysis it is occasionally useful to encode an operator (say, on $L^{2}(\mathbb{R})$ ) by its symbol. In the finite dimensional case, this corresponds to representing a linear transformation by a function on $\mathbb{Z}_{n} \times \hat{\mathbb{Z}}_{n}$. This is an entirely equivalent formulation-strictly speaking no information is either lost or gained. There will be situations in which such a symbol representation of an operator poses distinct advantages to the matrix representation.

As an application therefore we consider the cyclic group and give an associated symbol calculus for $n$ by $n$ matrices, culminating in a new decomposition of the Lie algebra $\operatorname{sl}(n)$ into a sum of Cartan subalgebras. 


\section{Notation and preliminaries}

For any finite set $S$, denote the space of complex-valued functions on $S$ by $C(S)$. If $S$ has measure $d s$, introduce the inner product $(\cdot, \cdot)$ on $C(S)$

$$
\left(f_{1}, f_{2}\right)=\int_{S} f_{1}(s) \overline{f_{2}(s)} d s, \quad \forall f_{1}, f_{2} \in C(G),
$$

and let $\delta_{s} \in C(S)$ be the function such that

$$
\int_{S} \delta_{s}\left(s^{\prime}\right) f\left(s^{\prime}\right) d s^{\prime}=f(s), \quad \forall f \in C(S) .
$$

If $S_{1}, S_{2}$ are sets and $\sigma: S_{1} \rightarrow S_{2}$ a map, let $a^{\sigma}=a \circ \sigma$ for all $a \in C\left(S_{2}\right)$. We let $\operatorname{Perm}(S)$ denote the group of permutations of the set $S$.

Now let $G$ be a finite abelian group with $|G|=n$ and with typical element $x$, operation addition and identity 0 . Let $\widehat{G}$ be the dual group with typical element $Y$ and denote the pairing between $x \in G$ and $Y \in \widehat{G}$ by $\langle Y, x\rangle$. Let $d x$ denote a Haar measure on $G$ (unique up to a constant).

The Fourier transform ${ }^{\wedge}: C(G) \rightarrow C(\widehat{G})$ is given by

$$
f^{\wedge}(Y)=\int_{G} \overline{\langle Y, x\rangle} f(x) d x, \quad \forall f \in C(G) .
$$

Normalize Haar measure $d Y$ on $\widehat{G}$ so that the inverse ${ }^{\vee}$ of the Fourier transform is given by

$$
h^{\vee}(x)=\int_{\widehat{G}}\langle Y, x\rangle h(Y) d Y, \quad \forall h \in C(\widehat{G}) .
$$

Then both the Fourier transform and its inverse are unitary operators. For $x \in G$, let $\hat{\hat{x}}$ denote the element of $C(\widehat{G})$ given by $\hat{\hat{x}}(Y)=\langle Y, x\rangle$. The map $x \rightarrow \hat{\hat{x}}$ provides us with a natural identification of $G$ and $\widehat{G}$. For a general locally compact abelian group, there is of course no isomorphism between $G$ and $\widehat{G}$ but when $G$ is finite there is, although it is not unique. This follows from the basic structure theorem that states that any finite abelian group is isomorphic to a direct product of cyclic groups along with the obvious fact that the dual of the cyclic group $\mathbb{Z}_{n}$ is itself isomorphic to $\mathbb{Z}_{n}$.

Let $\widetilde{G}=G \times \widehat{G}$ with typical element $z=(x, Y)$ and Haar measure $d z=d x d Y$. Note that this measure is canonical; it is independent of the original choice of $d x$. In fact, $\int_{\tilde{G}} d z=|G|=n$. Furthermore we find that with these normalizations $\delta_{z_{0}}(z)$ is the function of $z$ that is zero unless $z=z_{0}$, in which case it is $|G|=n$. From this also follows the useful formula

$$
\int_{\tilde{G}}\left\langle z, z_{1}\right\rangle d z=\delta_{0}\left(z_{1}\right)
$$


which we will use throughout. $\widetilde{G}$ also has the canonical function $e(x, Y)=\langle Y, x\rangle$. Now $(\widetilde{G})^{\Upsilon} \simeq \widehat{G} \times G$ with typical element $Z=(Y, x)$ and Haar measure $d Y d x$. Define $\tau:(\widetilde{G})^{\wedge} \rightarrow \widetilde{G}$ by $\tau(Y, x)=(x,-Y)$. Then $\tau$ is a canonical isomorphism from $(\tilde{G})$ to $\widetilde{G}$.

For $z_{i}=\left(x_{i}, Y_{i}\right) \in \widetilde{G}, i=1,2$, define $\left\langle z_{1}, z_{2}\right\rangle=\left\langle Y_{1}, x_{2}\right\rangle \overline{\left\langle Y_{2}, x_{1}\right\rangle}$. For $k>2$ and $z_{i}=\left(x_{i}, Y_{i}\right) \in \widetilde{G}, i=1, \ldots, k$, define $\left\langle z_{1}, \ldots, z_{k}\right\rangle=\left\langle z_{1}, z_{2}\right\rangle \cdots\left\langle z_{k-1}, z_{k}\right\rangle\left\langle z_{k}, z_{1}\right\rangle$. It is evident that for all $k \geq 2,\left\langle z_{1}, z_{2}, \ldots, z_{k}\right\rangle=\overline{\left\langle z_{k}, \ldots, z_{2}, z_{1}\right\rangle}$ and for $k>2$ the expression $\left\langle z_{1}, \ldots, z_{k}\right\rangle$ is invariant under cyclic permutations of the $z_{i}$ 's.

For $z \in \widetilde{G}$, let $\hat{z} \in C(\widetilde{G})$ be defined by $\hat{z}\left(z^{\prime}\right)=\left\langle z, z^{\prime}\right\rangle$ for all $z^{\prime} \in \widetilde{G}$.

\section{The *-product}

We will be interested in a particular algebra structure canonically defined on $C(\widetilde{G})$. For $a_{1}, a_{2} \in C(\widetilde{G})$, define $a_{1} * a_{2} \in C(\widetilde{G})$ by

$$
a_{1} * a_{2}\left(z_{3}\right)=\int_{\tilde{G}} \int_{\tilde{G}}\left\langle z_{1}, z_{2}, z_{3}\right\rangle a_{1}\left(z_{1}\right) a_{2}\left(z_{2}\right) d z_{1} d z_{2}, \quad \forall z_{3} \in \widetilde{G}
$$

THEOREM 3.1. ( $C(\widetilde{G}), *)$ is an associative algebra with identity the constant function 1.

Proof. Let $a_{1}, a_{2}, a_{3} \in C(\widetilde{G})$. Then for all $z_{4} \in \widetilde{G}$,

$$
\left(a_{1} * a_{2}\right) * a_{3}\left(z_{4}\right)=\int_{\widetilde{G}} \cdots \int_{\widetilde{G}}\left\langle z_{1}, z_{2}, z\right\rangle\left\langle z, z_{3}, z_{4}\right\rangle a_{1}\left(z_{1}\right) a_{2}\left(z_{2}\right) a_{3}\left(z_{3}\right) d z_{1} d z_{2} d z_{3} d z,
$$

while

$$
a_{1} *\left(a_{2} * a_{3}\right)\left(z_{4}\right)=\int_{\tilde{G}} \cdots \int_{\widetilde{G}}\left\langle z_{1}, z, z_{4}\right\rangle\left\langle z_{2}, z_{3}, z\right\rangle a_{1}\left(z_{1}\right) a_{2}\left(z_{2}\right) a_{3}\left(z_{3}\right) d z_{1} d z_{2} d z_{3} d z .
$$

Thus associativity of $*$ is equivalent to the validity for all $z_{1}, z_{2}, z_{3}, z_{4} \in \widetilde{G}$ of the equation

$$
\int_{\tilde{G}}\left\langle z_{1}, z_{2}, z\right\rangle\left\langle z, z_{3}, z_{4}\right\rangle d z=\int_{\tilde{G}}\left\langle z_{1}, z, z_{4}\right\rangle\left\langle z_{2}, z_{3}, z\right\rangle d z
$$

Now

$$
\begin{aligned}
\int_{\widetilde{G}}\left\langle z_{1}, z_{2}, z\right\rangle\left\langle z, z_{3}, z_{4}\right\rangle d z & =\int_{\widetilde{G}}\left\langle z_{1}, z_{2}\right\rangle\left\langle z_{3}, z_{4}\right\rangle\left\langle z, z_{1}-z_{2}+z_{3}-z_{4}\right\rangle d z \\
& =\delta_{z_{4}}\left(z_{1}-z_{2}+z_{3}\right)\left\langle z_{1}, z_{2}, z_{3}\right\rangle \\
& =\int_{\widetilde{G}}\left\langle z_{4}, z_{1}\right\rangle\left\langle z_{2}, z_{3}\right\rangle\left\langle z, z_{1}-z_{2}+z_{3}-z_{4}\right\rangle d z \\
& =\int_{\widetilde{G}}\left\langle z_{1}, z, z_{4}\right\rangle\left\langle z_{2}, z_{3}, z\right\rangle d z
\end{aligned}
$$


which proves associativity of $*$.

For $a \in C(\widetilde{G})$ and $z_{3} \in \widetilde{G}$,

$$
\begin{aligned}
a * 1\left(z_{3}\right) & =\int_{\widetilde{G}} \int_{\widetilde{G}}\left\langle z_{1}, z_{2}, z_{3}\right\rangle a\left(z_{1}\right) d z_{1} d z_{2} \\
& =\int_{\widetilde{G}} \int_{\widetilde{G}}\left\langle z_{2}, z_{3}-z_{1}\right\rangle\left\langle z_{3}, z_{1}\right\rangle a\left(z_{1}\right) d z_{1} d z_{2} \\
& =\int_{\widetilde{G}} \delta_{0}\left(z_{3}-z_{1}\right)\left\langle z_{3}, z_{1}\right\rangle a\left(z_{1}\right) d z_{1}=a\left(z_{3}\right) .
\end{aligned}
$$

Similarly $1 * a=a$ for all $a \in C(\widetilde{G})$.

The computation in the above theorem also shows the following.

PROPOSITION 3.2. For $z_{1}, z_{2}, z_{3} \in \widetilde{G}, \delta_{z_{1}} * \delta_{z_{2}} * \delta_{z_{3}}=\left\langle z_{1}, z_{2}, z_{3}\right\rangle \delta_{z_{4}}$, where $z_{4}=$ $z_{1}-z_{2}+z_{3}$.

LEMMA 3.3. For $a_{1}, a_{2} \in C(\widetilde{G}), a_{2} * a_{1}=\overline{\bar{a}_{1} * \bar{a}_{2}}$.

Proof. For $z_{3} \in \widetilde{G}$,

$$
\begin{aligned}
a_{2} * a_{1}\left(z_{3}\right) & =\int_{\tilde{G}} \int_{\tilde{G}}\left\langle z_{1}, z_{2}, z_{3}\right\rangle a_{2}\left(z_{1}\right) a_{1}\left(z_{2}\right) d z_{1} d z_{2} \\
& =\int_{\widetilde{G}} \int_{\widetilde{G}}\left\langle z_{2}, z_{1}, z_{3}\right\rangle a_{1}\left(z_{1}\right) a_{2}\left(z_{2}\right) d z_{1} d z_{2} \\
& =\int_{\tilde{G}} \int_{\widetilde{G}} \overline{\left\langle z_{1}, z_{2}, z_{3}\right\rangle} a_{1}\left(z_{1}\right) a_{2}\left(z_{2}\right) d z_{1} d z_{2}=\overline{\bar{a}_{1} * \bar{a}_{2}}\left(z_{3}\right) .
\end{aligned}
$$

LEMMA 3.4. If $a_{i} \in C(\widetilde{G})$ and $a_{i}(x, Y)=a_{i}(x), i=1,2$ then $a_{1} * a_{2}=a_{1} a_{2}$. The same holds if $a_{i}(x, Y)=a_{i}(Y), i=1,2$.

Proof. Suppose that $a_{i}(x, Y)=a_{i}(x)$. Then for $z_{3}=\left(x_{3}, Y_{3}\right) \in \widetilde{G}$,

$$
\begin{aligned}
a_{1} * a_{2}\left(z_{3}\right)= & \int_{G} \int_{\widehat{G}} \int_{G} \int_{\widehat{G}}\left\langle Y_{1}, x_{2}-x_{3}\right\rangle\left\langle Y_{2}, x_{3}-x_{1}\right\rangle\left\langle Y_{3}, x_{1}-x_{2}\right\rangle \\
& \times a_{1}\left(x_{1}\right) a_{2}\left(x_{2}\right) d Y_{1} d x_{1} d Y_{2} d x_{2} \\
= & \int_{G} \int_{G} \delta_{0}\left(x_{2}-x_{3}\right) \delta_{0}\left(x_{3}-x_{1}\right)\left\langle Y_{3}, x_{1}-x_{2}\right\rangle a_{1}\left(x_{1}\right) a_{2}\left(x_{2}\right) d x_{1} d x_{2} \\
= & a_{1}\left(x_{3}\right) a_{2}\left(x_{3}\right) .
\end{aligned}
$$

Proposition 3.5. For $z_{1}, z_{2} \in \widetilde{G}, \hat{z}_{1} * \hat{z}_{2}=\left\langle z_{2}, z_{1}\right\rangle\left(z_{1}+z_{2}\right)^{\wedge}$. 
PROOF.

$$
\begin{aligned}
\hat{z}_{1} * \hat{z}_{2}\left(z_{3}\right) & =\int_{\widetilde{G}} \int_{\widetilde{G}}\left\langle z_{1}^{\prime}, z_{2}^{\prime}, z_{3}\right\rangle\left\langle z_{1}, z_{1}^{\prime}\right\rangle\left\langle z_{2}, z_{2}^{\prime}\right\rangle d z_{1}^{\prime} d z_{2}^{\prime} \\
& =\int_{\widetilde{G}} \delta_{0}\left(z_{2}^{\prime}-z_{3}-z_{1}\right)\left\langle z_{2}^{\prime}, z_{3}\right\rangle\left\langle z_{2}, z_{2}^{\prime}\right\rangle d z_{2}^{\prime} \\
& =\left\langle z_{1}, z_{3}\right\rangle\left\langle z_{2}, z_{1}+z_{3}\right\rangle=\left\langle z_{2}, z_{1}\right\rangle\left(z_{1}+z_{2}\right)^{\wedge}\left(z_{3}\right)
\end{aligned}
$$

COROLlary 3.6. For $z \in \widetilde{G}$ and $a \in C(\widetilde{G})$,

(1) $\hat{z} * a\left(z^{\prime}\right)=\hat{z}\left(z^{\prime}\right) a\left(z^{\prime}+z\right)$;

(2) $a * \hat{z}\left(z^{\prime}\right)=\hat{z}\left(z^{\prime}\right) a\left(z^{\prime}-z\right)$ for all $z^{\prime} \in \widetilde{G}$.

Proof. Proposition 3.5 shows the formulae hold when $a=\hat{z}_{0}$ for $z_{0} \in \widetilde{G}$ and thus generally since $\left\{\hat{z}_{0} \mid z_{0} \in \widetilde{G}\right\}$ spans $C(\widetilde{G})$.

Proposition 3.5 provides us with a ready supply of subalgebras of $(C(\widetilde{G}), *)$, for if $H$ is any subgroup of $\widetilde{G}$ it follows that the span of $\{\hat{z} \mid z \in H\}$ is a subalgebra of $C(\widetilde{G})$.

PROPOSITION 3.7. For $a_{1}, a_{2} \in C(\widetilde{G}),\left(a_{1}, a_{2}\right)=\int_{\widetilde{G}} a_{1} * \bar{a}_{2}(z) d z$.

PROOF.

$$
\begin{aligned}
\int_{\widetilde{G}} a_{1} * \bar{a}_{2}(z) d z & =\int_{\widetilde{G}} \int_{\widetilde{G}} \int_{\widetilde{G}}\left\langle z_{1}, z_{2}\right\rangle\left\langle z_{2}, z\right\rangle\left\langle z, z_{1}\right\rangle a_{1}\left(z_{1}\right) \overline{a_{2}\left(z_{2}\right)} d z_{1} d z_{2} d z \\
& =\int_{\widetilde{G}} \int_{\widetilde{G}} \delta_{0}\left(z_{1}-z_{2}\right)\left\langle z_{1}, z_{2}\right\rangle a_{1}\left(z_{1}\right) \overline{a_{2}\left(z_{2}\right)} d z_{1} d z_{2} \\
& =\int_{\widetilde{G}} a_{1}\left(z_{1}\right) \overline{a_{2}\left(z_{1}\right)} d z_{1}=\left(a_{1}, a_{2}\right) .
\end{aligned}
$$

From Proposition 3.7 and Lemma 3.3 one easily obtains the following.

Proposition 3.8. For $a_{1}, a_{2}, a_{3} \in C(\widetilde{G})$,

(1) $\int_{\tilde{G}} a_{1} * a_{2}(z) d z=\int_{\tilde{G}} a_{2} * a_{1}(z) d z$;

(2) $\left(a_{1}, a_{2} * a_{3}\right)=\left(a_{1} * \vec{a}_{3}, a_{2}\right)$;

(3) $\left(a_{1} * a_{2}, a_{3}\right)=\left(a_{2}, \bar{a}_{1} * a_{3}\right)$.

There are a number of other algebra structures on $C(\widetilde{G})$ that can be derived from the *-product. Suppose for example that $\sigma$ is a permutation of $\widetilde{G}$. For $a_{1}, a_{2} \in C(\widetilde{G})$ define $a_{1} *_{\sigma} a_{2} \in C(\widetilde{G})$ by $a_{1} *_{\sigma} a_{2}=\left(a_{1}^{\sigma} * a_{2}^{\sigma}\right)^{\sigma^{-1}}$. Then for all $z_{3} \in \widetilde{G}$,

$$
a_{1} *_{\sigma} a_{2}\left(z_{3}\right)=\int_{\tilde{G}} \int_{\tilde{G}}\left\langle\sigma\left(z_{1}\right), \sigma\left(z_{2}\right), \sigma\left(z_{3}\right)\right\rangle a_{1}\left(z_{1}\right) a_{2}\left(z_{2}\right) d z_{1} d z_{2},
$$


and the algebra structure $*_{\sigma}$ is isomorphic to $*$. In particular if $k \in \mathbb{Z}$ and $k$ and $|G|=n$ are relatively prime, then the map $\sigma((x, Y))=(k x, Y)$ is a permutation of $\widetilde{G}$ and we have

$$
a_{1} *_{\sigma} a_{2}\left(z_{3}\right)=\int_{\widetilde{G}} \int_{\widetilde{G}}\left\langle z_{1}, z_{2}, z_{3}\right\rangle^{k} a_{1}\left(z_{1}\right) a_{2}\left(z_{2}\right) d z_{1} d z_{2} .
$$

In this case we will write $*_{\sigma}$ as $*_{k}$. Then $k$ plays the role that Planck's constant $\hbar$ plays in the theory of the $*$-product for $\mathbb{R}$. It is easily seen that the constant function 1 is the identity for all $*_{k}$ and that for $z_{1}, z_{2} \in \widetilde{G}$, Proposition 3.5 becomes

$$
\hat{z}_{1} *_{k} \hat{z}_{2}=\left\langle z_{2}, z_{1} / k\right\rangle\left(z_{1}+z_{2}\right)^{\wedge}=\left\langle z_{2} / k, z_{1}\right\rangle\left(z_{1}+z_{2}\right) \hat{\text {, }}
$$

where $z \rightarrow z / k$ is the inverse map to $z \rightarrow k z$. The case $k=2$ will be of special importance for what follows.

\section{The symbol calculus}

Define a locally compact abelian group $G$ to be odd if the map $x \rightarrow 2 x$ is an automorphism of $G$. Then for example $\mathbb{R}$ is odd while the unit circle $S^{1}$ is not. A finite abelian group $G$ is odd if and only if $|G|=n$ is odd. Throughout this section we will assume that the finite abelian group $G$ is odd, so that $x / 2$ is well-defined for all $x \in G$.

For $a \in C(\widetilde{G})$, define $W^{a}: C(G) \rightarrow C(G)$ by

$$
W^{a} f(x)=\int_{\widehat{G}} \int_{G}\left\langle Y^{\prime}, x^{\prime}-x\right) a\left(\left(x+x^{\prime}\right) / 2, Y^{\prime}\right) f\left(x^{\prime}\right) d x^{\prime} d Y^{\prime}
$$

for all $f \in C(G)$ and $x \in G$. This is essentially the same formula as used in $\mathbb{R}$ to define an operator from a 'Weyl' symbol. The following facts are immediate.

LEMMA 4.1. (1) If $a(x, Y)=a(x)$, then $W^{a}$ is multiplication by $a(x)$.

(2) If $a(x, Y)=a(Y)$ then $W^{a}$ is multiplication by $a(Y)$ on the Fourier transform side.

(3) If $a=1$ then $W^{a}$ is the identity.

PROPOSITION 4.2. For $a \in C(\widetilde{G})$ the adjoint of $W^{a}$ is $W^{\bar{a}}$.

Proof. Let $a \in C(\widetilde{G})$ and $f_{1}, f_{2} \in C(G)$. Then

$$
\begin{aligned}
\left(W^{a} f_{1}, f_{2}\right) & =\int_{G} \int_{\widehat{G}} \int_{G}\left\langle Y^{\prime}, x^{\prime}-x\right) a\left(\left(x+x^{\prime}\right) / 2, Y^{\prime}\right) f_{1}\left(x^{\prime}\right) \overline{f_{2}(x)} d x^{\prime} d Y^{\prime} d x \\
& =\int_{G} \int_{\widehat{G}} \int_{G} f_{1}\left(x^{\prime}\right) \overline{\left(Y^{\prime}, x-x^{\prime}\right) \bar{a}\left(\left(x+x^{\prime}\right) / 2, Y^{\prime}\right) f_{2}(x)} d x d Y^{\prime} d x^{\prime} \\
& =\left(f_{1}, W^{\bar{a}} f_{2}\right) .
\end{aligned}
$$


If $a \in C(\widetilde{G})$, then $a^{\tau} \in C(\widehat{G} \times G)$ so that reversing the roles of $G$ and $\widehat{G}$ in (4.1) we may regard $W^{a^{r}}$ as an operator from $C(\widehat{G})$ to $C(G)$.

Proposition 4.3. For $a \in C(\widetilde{G})$ let $b=a^{\tau^{-1}} \in C((\widetilde{G}))$. Then for all $f \in C(G)$, $\left(W^{a} f\right)^{\wedge}=W^{b} f^{\wedge}$.

PROOF.

$$
\begin{aligned}
\left(W^{a} f\right)^{\wedge}(Y) & =\int_{G} \int_{\widehat{G}} \int_{G} \overline{\langle Y, x\rangle}\left\langle Y^{\prime}, x^{\prime}-x\right\rangle a\left(\left(x+x^{\prime}\right) / 2, Y^{\prime}\right) f\left(x^{\prime}\right) d x^{\prime} d Y^{\prime} d x \\
& =\int_{G} \int_{\widehat{G}} \overline{\langle Y, x\rangle} \overline{\left\langle Y^{\prime}, x\right\rangle} a\left(x / 2, Y^{\prime}\right) f^{\wedge}\left(-2 Y^{\prime}-Y\right) d Y^{\prime} d x .
\end{aligned}
$$

Replace $Y^{\prime}$ with $\left(Y^{\prime}-Y\right) / 2$ to get

$$
\left(W^{a} f\right)^{\dot{\ddots}}(Y)=\int_{G} \int_{\widehat{G}} \frac{\vdots}{\left\langle\left(Y^{\prime}+Y\right) / 2, x\right\rangle} a\left(x / 2,\left(Y^{\prime}-Y\right) / 2\right) f^{\wedge}\left(Y^{\prime}\right) d Y^{\prime} d x .
$$

Finally replace $x$ with $2 x$ and $Y^{\prime}$ with $-Y^{\prime}$ to get

$$
\begin{aligned}
\left(W^{a} f\right)^{\wedge}(Y) & =\int_{G} \int_{\widehat{G}}\left\langle Y^{\prime}-Y, x\right\rangle a\left(x,-\left(Y^{\prime}+Y\right) / 2\right) f^{\wedge}\left(Y^{\prime}\right) d Y^{\prime} d x \\
& =W^{b}\left(f^{\wedge}\right)(Y) .
\end{aligned}
$$

Proposition 4.4. For $z=\left(x_{0}, Y_{0}\right) \in \widetilde{G}$ and $f \in C(G)$,

$$
W^{\hat{z}} f(x)=\left\langle Y_{0}, x_{0} / 2\right\rangle\left\langle Y_{0}, x\right\rangle f\left(x+x_{0}\right) \quad \forall x \in G .
$$

ProOf.

$$
\begin{aligned}
W^{\hat{z}} f(x) & =\int_{\widehat{G}} \int_{G}\left\langle Y^{\prime}, x^{\prime}-x\right\rangle\left\langle Y_{0},\left(x+x^{\prime}\right) / 2\right\rangle \overline{\left\langle Y^{\prime}, x_{0}\right\rangle} f\left(x^{\prime}\right) d x^{\prime} d Y^{\prime} \\
& =\int_{G} \delta_{0}\left(x^{\prime}-x-x_{0}\right)\left\langle Y_{0},\left(x+x^{\prime}\right) / 2\right\rangle f\left(x^{\prime}\right) d x^{\prime} \\
& =\left\langle Y_{0}, x\right\rangle\left\langle Y_{0}, x_{0} / 2\right\rangle f\left(x+x_{0}\right) .
\end{aligned}
$$

We will be interested in recovering $a \in C(\widetilde{G})$ from a knowledge of $W^{a}$. For $Y \in \widehat{G}$ and $x \in G$,

$$
\begin{aligned}
W^{a} Y(x) & =\int_{\widehat{G}} \int_{G}\left\langle Y^{\prime}, x^{\prime}-x\right\rangle a\left(\left(x+x^{\prime}\right) / 2, Y^{\prime}\right)\left\langle Y, x^{\prime}\right\rangle d x^{\prime} d Y^{\prime} \\
& =\int_{\widehat{G}} \int_{G}\left\langle Y^{\prime}, x^{\prime}-2 x\right\rangle a\left(x^{\prime} / 2, Y^{\prime}\right)\left\langle Y, x^{\prime}-x\right\rangle d x^{\prime} d Y^{\prime} \\
& =\overline{\langle Y, x\rangle} \int_{\widehat{G}} \int_{G}\left\langle Y^{\prime}, 2 x^{\prime}-2 x\right\rangle\left\langle Y, 2 x^{\prime}\right\rangle a\left(x^{\prime}, Y^{\prime}\right) d x^{\prime} d Y^{\prime}
\end{aligned}
$$


Then if we define $c_{a} \in C(\tilde{G})$ by $c_{a}(z)=c_{a}(x, Y)=W^{a} Y(x)$ then

$$
c_{a}(z)=\overline{e(z)} \int_{\widetilde{G}}\left\langle z^{\prime},-2 z\right\rangle\left(e^{2} a\right)\left(z^{\prime}\right) d z^{\prime} .
$$

Taking the Fourier transform gives

$$
e^{2} a\left(z^{\prime}\right)=\int_{\tilde{G}}\left\langle 2 z^{\prime}, z\right\rangle\left(e c_{a}\right)(z) d z .
$$

We have proven the following.

Proposition 4.5. Let $T: C(G) \rightarrow C(G)$ be any linear operator. Define $d_{T} \in$ $C(\widetilde{G})$ by $d_{T}(x, Y)=T Y(x)$. Then $T=W^{a}$, where

$$
a(z)=\bar{e}^{2}(z) \int_{\widetilde{G}}\left\langle 2 z, z^{\prime}\right\rangle e\left(z^{\prime}\right) d_{T}\left(z^{\prime}\right) d z^{\prime} .
$$

COROLlaRY 4.6. The map $a \rightarrow W^{a}$ is a linear isomorphism from $C(\tilde{G})$ to $\operatorname{End}(C(G))$.

We now relate the *-product to the algebra structure on $C(\widetilde{G})$ arising from its identification with End $(C(G))$.

THEOREM 4.7. For $a, b \in C(\widetilde{G})$, suppose $W^{a} W^{b}=W^{c}, c \in C(\widetilde{G})$. Then $c=$ $a *_{2} b$.

PROOF. For $(x, Y) \in \widetilde{G}$,

$$
\begin{aligned}
W^{a} W^{b} Y(x)= & \int_{\widehat{G}} \int_{G} \int_{\widehat{G}} \int_{G}\left\langle Y^{\prime}, x^{\prime}-x\right\rangle a\left(\left(x+x^{\prime}\right) / 2, Y^{\prime}\right)\left\langle Y^{\prime \prime}, x^{\prime \prime}-x^{\prime}\right\rangle \\
& \times b\left(\left(x^{\prime}+x^{\prime \prime}\right) / 2, Y^{\prime \prime}\right)\left\langle Y, x^{\prime \prime}\right\rangle d x^{\prime \prime} d Y^{\prime \prime} d x^{\prime} d Y^{\prime}
\end{aligned}
$$

Then from Proposition 4.5, for $\left(x_{1}, Y_{1}\right) \in \widetilde{G}$,

$$
\begin{aligned}
\left\langle Y_{1}, x_{1}\right)^{2} c\left(x_{1}, Y_{1}\right)= & \int_{\widehat{G}} \ldots \int_{G}\left\langle 2 Y_{1}, x\right\rangle \overline{\left\langle 2 Y, x_{1}\right\rangle}\langle Y, x\rangle\left\langle Y^{\prime}, x^{\prime}-x\right\rangle\left\langle Y^{\prime \prime}, x^{\prime \prime}-x^{\prime}\right\rangle\left\langle Y, x^{\prime \prime}\right\rangle \\
& \times a\left(\left(x+x^{\prime}\right) / 2, Y^{\prime}\right) b\left(\left(x^{\prime}+x^{\prime \prime}\right) / 2, Y^{\prime \prime}\right) d x^{\prime \prime} d Y^{\prime \prime} d x^{\prime} d Y^{\prime} d x d Y .
\end{aligned}
$$

Replace $x^{\prime \prime}$ with $x^{\prime \prime}-x^{\prime}$ to get

$$
\begin{aligned}
& \left\langle Y_{1}, x_{1}\right\rangle^{2} c\left(x_{1}, Y_{1}\right) \\
& =\int_{\widehat{G}} \ldots \int_{G}\left\langle 2 Y_{1}, x\right\rangle \overline{\left\langle 2 Y, x_{1}\right\rangle}\langle Y, x\rangle\left\langle Y^{\prime}, x^{\prime}-x\right\rangle\left\langle Y^{\prime \prime}, x^{\prime \prime}-2 x^{\prime}\right\rangle\left\langle Y, x^{\prime \prime}-x^{\prime}\right\rangle \\
& \quad \times a\left(\left(x+x^{\prime}\right) / 2, Y^{\prime}\right) b\left(x^{\prime \prime} / 2, Y^{\prime \prime}\right) d x^{\prime \prime} d Y^{\prime \prime} d x^{\prime} d Y^{\prime} d x d Y .
\end{aligned}
$$


Replace $x^{\prime}$ with $x^{\prime}-x$ to get

$$
\begin{aligned}
\left\langle Y_{1}, x_{1}\right\rangle^{2} c\left(x_{1}, Y_{1}\right) & \\
= & \int_{\widehat{G}} \ldots \int_{G}\left\langle 2 Y_{1}, x\right\rangle \overline{\left\langle 2 Y, x_{1}\right\rangle}\langle Y, x\rangle\left\langle Y^{\prime}, x^{\prime}-2 x\right\rangle\left\langle Y^{\prime \prime}, x^{\prime \prime}-2 x^{\prime}+2 x\right\rangle \\
& \times\left\langle Y, x^{\prime \prime}-x^{\prime}+x\right\rangle a\left(x^{\prime} / 2, Y^{\prime}\right) b\left(x^{\prime \prime} / 2, Y^{\prime \prime}\right) d x^{\prime \prime} d Y^{\prime \prime} d x^{\prime} d Y^{\prime} d x d Y \\
= & \int_{\widehat{G}} \ldots \int_{G}\left\langle 2 Y_{1}, x\right\rangle \overline{\left\langle 2 Y, x_{1}\right\rangle}\langle Y, x\rangle\left\langle Y^{\prime}, 2 x^{\prime}-2 x\right\rangle\left\langle Y^{\prime \prime}, 2 x^{\prime \prime}-4 x^{\prime}+2 x\right\rangle \\
& \times\left\langle Y, 2 x^{\prime \prime}-2 x^{\prime}+x\right\rangle a\left(x^{\prime}, Y^{\prime}\right) b\left(x^{\prime \prime}, Y^{\prime \prime}\right) d x d Y d x^{\prime} d Y^{\prime} d x^{\prime \prime} d Y^{\prime \prime} .
\end{aligned}
$$

After integrating with respect to $Y$ and $x$ and cancelling terms, we obtain

$$
\begin{aligned}
c\left(x_{1}, Y_{1}\right)= & \int_{\widehat{G}} \int_{G} \int_{\widehat{G}} \int_{G}\left\langle 2 Y_{1}, x^{\prime}-x^{\prime \prime}\right\rangle\left\langle 2 Y^{\prime}, x^{\prime \prime}-x_{1}\right\rangle\left\langle 2 Y^{\prime \prime}, x_{1}-x^{\prime}\right\rangle \\
& \times a\left(x^{\prime}, Y^{\prime}\right) b\left(x^{\prime \prime}, Y^{\prime \prime}\right) d x^{\prime} d Y^{\prime} d x^{\prime \prime} d Y^{\prime \prime}=a *_{2} b\left(x_{1}, Y_{1}\right) .
\end{aligned}
$$

COROLLARY 4.8. If $G$ is an odd finite abelian group, then $(C(\widetilde{G}), *)$ is isomorphic to the algebra of $n \times n$ matrices.

Proof. This follows from Theorem 4.7 and the observation in Section 3 that if $G$ is odd, then $(C(\widetilde{G}), *)$ and $\left(C(\widetilde{G}), *_{2}\right)$ are isomorphic.

For a general abelian group $G,(C(\widetilde{G}), *)$ is isomorphic to a direct sum of matrix algebras (this actually follows from the results of Section 3). However if $G$ is not odd there will generally be more than one component in this sum. For example if $G=\mathbb{Z}_{2} \times \cdots \times \mathbb{Z}_{2}$ then one may check directly that $(C(\widetilde{G}), *)$ is a direct sum of 1-dimensional algebras, and therefore abelian.

\section{The Heisenberg group}

Throughout this section $G$ will be an odd finite abelian group. Let $S_{G}$ be the subset of the unit circle $S^{1} \subseteq \mathbb{C}$ given by $S_{G}=\{\langle Y, x\rangle \mid Y \in \widehat{G}, x \in G\}$.

LEMMA 5.1. $S_{G}$ is a subgroup of $S^{1}$.

PRoOF. Suppose that $G \simeq \mathbb{Z}_{n_{1}} \times \cdots \times \mathbb{Z}_{n_{i}}$. Then $S_{G}$ consists of all complex numbers of the form $\gamma_{1} \cdots \gamma_{l}$ with $\gamma_{i}$ an $n_{i}$ th root of unity. This is clearly a subgroup of $S^{1}$.

Any finite subgroup of $S^{1}$ is cyclic. Let $\left|S_{G}\right|=m$, so that $S_{G}$ consists of all $m$ th roots of unity. If $G \simeq \mathbb{Z}_{n_{1}} \times \cdots \times \mathbb{Z}_{n_{i}}$ then $m$ is the least common multiple of the $n_{i}$ 's, $i=1, \ldots, l$. It follows that for any $k \in \mathbb{Z},(k, m)=1$ if and only if $(k, n)=1$. 
Let $H_{G}=\widetilde{G} \times S_{G}$ and introduce a multiplication in $H_{G}$ by

$$
\left(z_{1}, \gamma_{1}\right) \cdot\left(z_{2}, \gamma_{2}\right)=\left(z_{1}+z_{2}, \gamma_{1} \gamma_{2}\left\langle z_{2} / 2, z_{1}\right\rangle\right)
$$

Then $H_{G}$ is a finite nilpotent group with identity $(0,1)$ and centre

$$
Z_{G}=\left\{(0, \gamma) \mid \gamma \in S_{G}\right\} \simeq S_{G}
$$

We call $H_{G}$ the Heisenberg group of $G$.

Suppose that $\rho$ is any irreducible unitary representation of $H_{G}$. Then $Z_{G}$ acts as scalars so there is a character $\chi$ of $Z_{G}$ such that $\rho(\gamma)=\chi(\gamma) I$ for all $\gamma \in Z_{G}$. Since any character $\chi$ of $Z_{G} \simeq S_{G}$ is given by $\chi(\gamma)=\gamma^{k}$ for some $k \in \mathbb{Z}, 0 \leq k<m$, we may separate the unitary dual $\left(H_{G}\right)$ of $H_{G}$ into $m$ components

$$
\left(H_{G}\right)=\bigcup_{k=0}^{m-1}\left(H_{G} \hat{)_{k}}\right.
$$

with $\rho \in\left(H_{G}\right)_{k}$ if and only if the central character of $\rho$ is $\gamma \rightarrow \gamma^{k}$.

For $0 \leq k<m$, let

$$
C\left(H_{G}\right)_{k}=\left\{\phi \in C\left(H_{G}\right) \mid \phi(\gamma h)=\gamma^{k} \phi(h) \forall \gamma \in Z_{G}, h \in H_{G}\right\} .
$$

Then we have the orthogonal decomposition $C\left(H_{G}\right)=\bigoplus_{k=0}^{m-1} C\left(H_{G}\right)_{k}$ and $\rho \in\left(H_{G}\right)_{k}$ if and only if all the matrix coefficients of $\rho$ belong to $C\left(H_{G}\right)_{k}$ (recall that a matrix coefficient of $\rho$ is a function on $H_{G}$ of the form $h \rightarrow(\rho(h) v, w)$, where $v, w$ are vectors in the space of $\rho$ ).

PROPOSITION 5.2. There is (up to equivalence) exactly one representation in $\left(H_{G}\right)_{1}$. It acts on $C(G)$ and is given by $\rho_{1}(z, \gamma)=\gamma W^{\hat{z}}$.

Proof. It is easy to check that $\rho_{1}$ is a unitary representation of $H_{G}$. Since $\{\hat{z} \mid z \in \widetilde{G}\}$ spans $C(\widetilde{G})$ the representation is irreducible and clearly belongs to $\left(H_{G}\right)_{1}$. Now since the space of matrix coefficients of $\rho_{1}$ is $n^{2}$ dimensional it exhausts $C\left(H_{G}\right)_{1}$. Thus there are no other representations in $\left(H_{G}\right)_{1}$.

\section{The symplectic group and the oscillator representation}

$\widetilde{G}$ is a finite abelian group with additional structure determined by the 'form' $\langle\cdot, \cdot\rangle$. Let $\operatorname{Hom}(\widetilde{G})$ denote the ring of homomorphisms $\sigma: \widetilde{G} \rightarrow \widetilde{G}$, and $\operatorname{Aut}(\widetilde{G})$ the subgroup of automorphisms. For any $\sigma \in \operatorname{Aut}(\widetilde{G})$, there is a unique $\sigma^{*} \in \operatorname{Aut}(G)$ such that $\left\langle\sigma^{*} z_{1}, z_{2}\right\rangle=\left\langle z_{1}, \sigma z_{2}\right\rangle$ for all $z_{1}, z_{2} \in \widetilde{G}$. In analogy with the case $G=\mathbb{R}$ we define 
the symplectic group $\operatorname{Sp}(\widetilde{G})$ to be the set of automorphisms $\sigma$ of $\widetilde{G}$ which preserve $\langle\cdot, \cdot\rangle$, that is the set of $\sigma \in \operatorname{Aut}(\widetilde{G})$ such that $\sigma^{*}=\sigma^{-1}$. It follows immediately that for $\sigma \in \operatorname{Sp}(\widetilde{G})$,

$$
a^{\sigma} * b^{\sigma}=(a * b)^{\sigma}, \quad \forall a, b \in C(\widetilde{G}),
$$

so that $*_{\sigma}=*$.

Let $\operatorname{Perm}(\widetilde{G}, *)$ denote the group of permutations $\sigma$ of $\widetilde{G}$ which satisfy (6.1). Clearly $\operatorname{Sp}(\widetilde{G})$ is a subgroup of $\operatorname{Perm}(\widetilde{G}, *)$ and we may consider $\widetilde{G}$ as contained in $\operatorname{Perm}(\widetilde{G}, *)$ if we identify $z \in \widetilde{G}$ with the permutation $\sigma_{z}$ of $\widetilde{G}$ given by

$$
\sigma_{z}\left(z^{\prime}\right)=z^{\prime}+z, \quad \forall z^{\prime} \in \widetilde{G} .
$$

THEOREM 6.1. $\operatorname{Perm}(\widetilde{G}, *)=\widetilde{G} \times \operatorname{Sp}(\widetilde{G})$.

ProOF. $\widetilde{G} \cap \operatorname{Sp}(\widetilde{G})$ contains just the identity and $\operatorname{Sp}(\widetilde{G})$ normalizes $\widetilde{G}$. It thus suffices to show that if $\sigma \in \operatorname{Perm}(\widetilde{G}, *)$ and $\sigma(0)=0$, then $\sigma \in \operatorname{Sp}(\widetilde{G})$.

First note that (6.1) is equivalent to the condition

$$
\left\langle\sigma z_{1}, \sigma z_{2}, \sigma z_{3}\right\rangle=\left\langle z_{1}, z_{2}, z_{3}\right\rangle, \quad \forall z_{1}, z_{2}, z_{3} \in \widetilde{G} .
$$

Setting $z_{3}=0$ and using the condition $\sigma(0)=0$ shows that

$$
\left\langle\sigma z_{1}, \sigma z_{2}\right\rangle=\left\langle z_{1}, z_{2}\right\rangle, \quad \forall z_{1}, z_{2} \in \widetilde{G} .
$$

We thus need only show that $\sigma$ is a homomorphism. Recall that every character of $\widetilde{G}$ is of the form $\hat{z}$ for some $z \in \widetilde{G}$. Then the computation

$$
\begin{aligned}
(\hat{z})^{\sigma}\left(z^{\prime}\right) & =\hat{z}\left(\sigma z^{\prime}\right)=\left\langle z, \sigma z^{\prime}\right\rangle \\
& =\left\langle\sigma^{-1} z, z^{\prime}\right\rangle=\left(\sigma^{-1} z\right)^{\wedge}\left(z^{\prime}\right), \quad \forall z^{\prime} \in \widetilde{G}
\end{aligned}
$$

shows that the map $\chi \rightarrow \chi^{\sigma}$ permutes the characters of $\widetilde{G}$.

Now let $z_{1}, z_{2}, z_{3} \in \widetilde{G}$. Then since characters separate points of an abelian group,

$$
\begin{aligned}
z_{1} z_{2}=z_{3} & \Longleftrightarrow \chi\left(z_{1}\right) \chi\left(z_{2}\right)=\chi\left(z_{3}\right), & & \forall \chi \in(\widetilde{G}), \\
& \Longleftrightarrow \chi^{\sigma}\left(z_{1}\right) \chi^{\sigma}\left(z_{2}\right)=\chi^{\sigma}\left(z_{3}\right), & & \forall \chi \in(\widetilde{G}), \\
& \Longleftrightarrow \chi\left(\sigma z_{1}\right) \chi\left(\sigma z_{2}\right)=\chi\left(\sigma z_{3}\right), & & \forall \chi \in(\widetilde{G}), \\
& \Longleftrightarrow\left(\sigma z_{1}\right)\left(\sigma z_{2}\right)=\sigma z_{3} . & &
\end{aligned}
$$

If $\sigma \in \operatorname{Perm}(\widetilde{G}, *)$ then Proposition 3.7 shows that $\sigma$ preserves the inner product $(\cdot, \cdot)$ on $C(\widetilde{G})$. Now suppose that $G$ is odd. Then $\operatorname{Perm}(\widetilde{G}, *)$ acts as a group of automorphisms of the Hilbert algebra $\left(C(\widetilde{G}), *_{2}\right)$ which we have seen is isomorphic 
to $M(n, \mathbb{C})$. Now any automorphism of $M(n, \mathbb{C})$ which preserves the Hilbert space structure must be conjugation by a unitary matrix. Thus for each $\mu \in \operatorname{Perm}(\widetilde{G}, *)$ we can find $c_{\mu} \in C(\widetilde{G})$ such that for all $b \in C(\widetilde{G})$

$$
c_{\mu} *_{2} b *_{2} \bar{c}_{\mu}(z)=b(\mu(z)), \quad \forall z \in \widetilde{G} .
$$

Furthermore $c_{\mu}$ is unique up to a multiplicative constant of modulus one. We may restate this by saying that $\mu \rightarrow W^{c_{\mu}}$ is a projective unitary representation of $\operatorname{Perm}(\widetilde{G}, *)$ which we call the oscillator representation. For $\mu \in \operatorname{Sp}(\widetilde{G})$, the correspondence $\mu \rightarrow c_{\mu}$ involves a 'Cayley transform'.

THEOREM 6.2. Suppose $G$ is odd. Let $\mu \in \operatorname{Sp}(\widetilde{G})$ and suppose that $1+\mu \epsilon$ $\operatorname{Hom}(\widetilde{G})$ is invertible. Define $C(\mu)=(1-\mu) /(1+\mu)$. Then $c_{\mu}(z)=\langle z, C(\mu) z\rangle$.

Proof. For $\sigma \in \operatorname{Hom}(\widetilde{G})$, set $a_{\sigma}(z)=\langle z, \sigma z\rangle$ for all $z \in \widetilde{G}$. Then for $b \in C(\widetilde{G})$,

$$
\begin{aligned}
a_{\sigma} *_{2} b *_{2} \bar{a}_{\sigma}(z)= & \int_{\widetilde{G}} \int_{\widetilde{G}} \int_{\widetilde{G}} \int_{\widetilde{G}}\left\langle z_{1}, z_{2}\right\rangle^{2}\left\langle z_{2}, z_{3}\right\rangle^{2}\left\langle z_{3}, z_{1}\right\rangle^{2}\left\langle z_{1}, \sigma z_{1}\right\rangle b\left(z_{2}\right) \\
& \times\left\langle z_{3}, z_{4}\right\rangle^{2}\left\langle z_{4}, z\right\rangle^{2}\left\langle z, z_{3}\right\rangle^{2}\left\langle\sigma z_{4}, z_{4}\right\rangle d z_{1} d z_{2} d z_{3} d z_{4} \\
= & \int_{\widetilde{G}} \int_{\widetilde{G}} \int_{\widetilde{G}}\left\langle z_{1}, z_{2}\right\rangle^{2} \delta_{0}\left(z_{1}-z_{2}+z_{4}-z\right)\left\langle z_{1}, \sigma z_{1}\right\rangle b\left(z_{2}\right) \\
& \times\left\langle z_{4}, z\right\rangle^{2}\left\langle\sigma z_{4}, z_{4}\right\rangle d z_{1} d z_{2} d z_{4} \\
= & \int_{\widetilde{G}} \int_{\widetilde{G}}\left\langle z_{1}, z_{4}-z\right\rangle^{2}\left\langle z_{1}, \sigma z_{1}\right\rangle b\left(z_{1}+z_{4}-z\right)\left\langle z_{4}, z\right\rangle^{2} \\
& \times\left\langle\sigma z_{4}, z_{4}\right\rangle d z_{1} d z_{4}
\end{aligned}
$$

Now make the change of variables $t=z_{1}+z_{4}$ and $s=z_{1}-z_{4}$ so that

$$
z_{1}=(t+s) / 2, \quad z_{4}=(t-s) / 2 .
$$

The above integral then becomes

$$
\begin{aligned}
\int_{\tilde{G}} \int_{\tilde{G}}\langle z, s\rangle^{2}\left\langle\frac{t+s}{2}, \frac{t-s}{2}\right\rangle^{2}\left\langle\frac{t+s}{2}, \sigma\left(\frac{t+s}{2}\right)\right\rangle \\
\quad \times\left\langle\sigma\left(\frac{t-s}{2}\right), \frac{t-s}{2}\right\rangle b(t-z) d t d s \\
=\int_{\tilde{G}} \int_{\tilde{G}}\langle z, s\rangle^{2}\langle s, t\rangle\left\langle t, \frac{\sigma s}{2}\right\rangle\left\langle s, \frac{\sigma t}{2}\right\rangle b(t-z) d t d s \\
=\int_{\tilde{G}} \delta_{0}\left(t-2 z-\frac{\sigma^{*} t}{2}+\frac{\sigma t}{2}\right) b(t-z) d t
\end{aligned}
$$


Assume for the moment that $2-\sigma^{*}+\sigma: \widetilde{G} \rightarrow \widetilde{G}$ is invertible. Then we may solve

$$
t=\left(2-\sigma^{*}+\sigma\right)^{-1}(4 z)
$$

so that the above integral becomes simply

$$
b\left(\left(2-\sigma^{*}+\sigma\right)^{-1}(4 z)-z\right)=b(\tau(z)),
$$

where

$$
\alpha(z)=\frac{2+\sigma^{*}-\sigma}{2-\sigma^{*}+\sigma}(z)=\frac{1-\left(\sigma-\sigma^{*}\right) / 2}{1+\left(\sigma-\sigma^{*}\right) / 2}(z) .
$$

We have established that if $\sigma \in \operatorname{Hom}(\widetilde{G})$ with $2-\sigma^{*}+\sigma$ invertible, then for all $b \in C(\widetilde{G}), a_{\sigma} *_{2} b *_{2} \bar{a}_{\sigma}(z)=b(\alpha(z))$, where $\alpha$ is defined in terms of $\sigma$ above.

Now suppose that $\mu \in S p(\widetilde{G})$ and that $1+\mu$ is invertible. Let $\eta=(1-\mu) /(1+\mu)$. Then $\eta^{*}=-\eta$. To see this, let $z_{1}, z_{2} \in \widetilde{G}$ with $z_{i}=(1+\mu) w_{i}$. Then

$$
\left\langle\eta z_{1}, z_{2}\right\rangle=\left\langle(1-\mu) w_{1},(1+\mu) w_{2}\right\rangle=\left\langle w_{1}, \mu w_{2}\right\rangle\left\langle\overline{\left.\mu w_{1}, w_{2}\right\rangle}\right.
$$

and similarly $\left\langle z_{1}, \eta z_{2}\right\rangle=\left\langle\mu w_{1}, w_{2}\right\rangle\left\langle\overline{w_{1}, \mu w_{2}}\right\rangle$ so the claim follows.

Consequently $2-\eta^{*}+\eta=2+2 \eta=4 /(1+\mu)$ is invertible, so replacing $\sigma$ in ( ) with $\eta$ we get that for all $b \in C(\widetilde{G}), c_{\mu} *_{2} b *_{2} \bar{c}_{\mu}(z)=b(\alpha(z))$, where $\alpha(z)=(1-\eta) /(1+\eta)=\mu(z)$.

\section{Systems of Cartan subalgebras for $\operatorname{sl}(n)$}

We now give some applications of our approach of regarding matrices from a symbol point of view. To be very concrete, we work out some of the ramifications of the preceding approach in the simplest possible case, that when $G=\mathbb{Z}_{3}=\{0,1,2\}$. The dual group $\widehat{G}$ can also be regarded as $\mathbb{Z}_{3}$, where the pairing between $k \in G$ and $l \in \widehat{G}$ is $\langle k, l\rangle=\xi^{k l}$ where $\xi=e^{2 \pi i / 3}$. Writing

$$
\widetilde{G}=G \times \widehat{G}=\left\{z_{k l}=(k, l) \mid 0 \leq k, l \leq 2\right\}
$$

we get $\left\langle z_{i j}, z_{k l}\right\rangle=\xi^{j k-i l}$. The space $V=C(G)$ has basis $\left\{e_{0}, e_{1}, e_{2}\right\}$ where $e_{i}(j)=\delta_{i j}$. We get from Proposition 4.4 the formula

$$
W^{\hat{z}_{i}} e_{l}(k)=\langle j, i / 2\rangle\langle j, k\rangle \delta_{l, k+i}=\xi^{2 i j} \xi^{j k(l-i)} \delta_{l-i, k}=\xi^{j(i+l)} e_{l-i}(k) .
$$

Thus $W^{\hat{z}_{i j}} e_{l}=\xi^{j(i+l)} e_{l-i}$, where of course the subscripts are computed mod 3. We will write $W^{\hat{z}_{i j}}=W_{i j}$, an operator on $V$ and let $M_{i j}$ denote the matrix of the operator 
in the basis $\left\{e_{0}, e_{1}, e_{2}\right\}$. Here are these nine matrices:

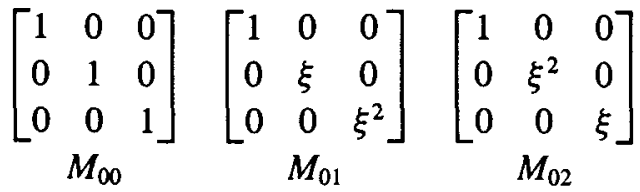

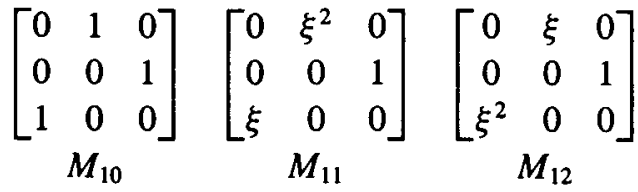

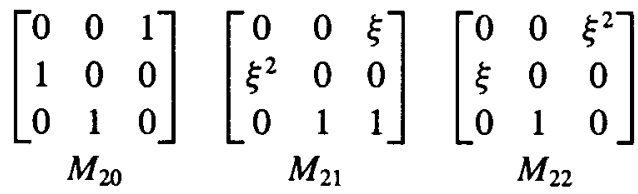

We now record various properties of these matrices which follow from Section 6 .

THEOREM 7.1. (1) $B=\left\{M_{i j} \mid 0 \leq i, j \leq 2\right\}$ forms a basis of $M(3, \mathbb{C})$.

(2) $B_{0}=B \backslash M_{00}$ forms a basis of $\mathrm{sl}(3, \mathbb{C})$, the trace zero $3 \times 3$ matrices.

(3) Each $M_{i j}$ is invertible, in fact unitary. Furthermore $M_{i j}^{-1}=M_{-i-j}$.

(4) $M_{i j} M_{k l}=\xi^{2(i l-j k)} M_{i+k, j+l}$.

(5) $M_{i j} M_{k l} M_{i j}^{-1}=\xi^{i l-j k} M_{k l}$.

(6) $M_{i j}^{T}=M_{-i, j}$.

(7) $\overline{M_{i j}}=M_{i,-j}$.

(8) If we define $M_{i j}^{\circ}=M_{j i}$ and extend to the entire algebra linearly, then $(A B)^{\circ}=$ $B^{\circ} A^{\circ}$.

Some remarks are in order. We will shortly see that Property (2) is potentially useful in Lie theory, where the fact that there is no 'canonical' basis of $\operatorname{sl}(n, \mathbb{C})$ constructed from the usual basis $E_{i j}$ (the matrix with a single 1 in the $i j$ th position) is always a source of some awkwardness. Property (4) show that pairwise the $M_{i j}$ 'almost' commute. Property (5) shows that conjugation by $M_{i j}$ is diagonalized by the subspaces $\left\langle M_{k l}\right\rangle$. Properties (6) and (7) reveal a symmetry between the operations of transposition and conjugation not so apparent in the usual formulation. Property (8) reveals another transposition-like symmetry which is canonical in this basis.

We now point out a consequence of our discussions of subalgebras of $C(\widetilde{G})$. There are 4 subgroups of $\widetilde{G}$ of size 3 , namely $G_{1}=\{(00),(01),(02)\}, G_{2}=$ $\{(00),(11),(22)\}, G_{3}=\{(00),(21),(12)\}$ and $G_{4}=\{(00),(10),(20)\}$. To each corresponds a 3-dimensional commutative subalgebra of $C(\widetilde{G}) \cong M(3, \mathbb{C})$, spanned by the corresponding matrices $M_{i j}$. When we pass to $\mathfrak{g}=\mathrm{sl}(3, \mathbb{C})$ regarded as a Lie 
algebra, we get the decomposition

$$
\mathfrak{g}=\mathfrak{h}_{1} \oplus \mathfrak{h}_{2} \oplus \mathfrak{h}_{3} \oplus \mathfrak{h}_{4},
$$

where $\mathfrak{h}_{1}=\left\langle M_{01}, M_{02}\right\rangle, \mathfrak{h}_{2}=\left\langle M_{11}, M_{22}\right\rangle, \mathfrak{h}_{3}=\left\langle M_{21}, M_{12}\right\rangle$ and $\mathfrak{h}_{4}=\left\langle M_{10}, M_{20}\right\rangle$.

This implies an interesting decomposition on the group level as well. If $H_{i}$ is the Cartan subgroup in $G=\operatorname{SL}(3, \mathbb{C})$ corresponding to $\mathfrak{h}_{i}$, then it is natural to ask to what extent does (*) extend to a decomposition of the form $\mathrm{G}=\mathrm{H}_{1} \mathrm{H}_{2} \mathrm{H}_{3} \mathrm{H}_{4}$ ? It will certainly hold in a neighbourhood of the identity.

The discussion here easily generalizes to $\operatorname{sl}(n)$. To what extent can we decompose other simple Lie algebras as direct sums of Cartan subalgebras?

\section{References}

[1] R. Aldrovandi and L. A. Saeger, 'Projective Fourier duality and Weyl quantization', Internat. J. Theoret. Phys. 36 (1997), 573-612.

[2] F. Bayen, M. Flato, C. Fronsdal, A. Lichnerowicz and D. Sternheimer, 'Deformation theory and quantization', Ann. Physics 111 (1978), 61-110, 111-151.

[3] G. Folland, Harmonic analysis on phase space (Princeton University Press, Princeton, NJ, 1989).

[4] A. Grossman, G. Loupias and E. M. Stein, 'An algebra of pseudo-differential operators and Quantum Mechanics in phase space', Ann. Inst. Fourier (Grenoble) 18 (1968), 343-368.

[5] L. Hormander, 'The Weyl calculus of pseudo-differential operators', Comm. Pure Appl. Math. 32 (1979), 127-208.

[6] R. Howe, 'Quantum Mechanics and partial differential equations', J. Funct. Anal. 38 (1980), 188-254.

[7] — 'The oscillator semigroup', in: The mathematical heritage of Hermann Weyl (Durham, NC, 1987), Proc. Sympos. Pure Math. 48 (Amer. Math. Soc., Providence, RI, 1988) pp. 61-132.

[8] G. Lion and M. Vergne, The Weil representation, Maslov index, and theta series (Birkhauser, Boston, 1980).

[9] J. E. Moyal, 'Quantum mechanics as a statistical theory', Math. Proc. Cambridge Philos. Soc. 45 (1949), 99-124.

[10] A. Weil, 'Sur certaines groupes d'opérateurs unitaires', Acta. Math. 111 (1964), 143-211.

\section{School of Mathematics}

UNSW

Sydney 2052

Australia

e-mail: n.wildberger@unsw.edu.au 